\title{
Pullback Exponential Attractors for Nonautonomous Reaction Diffusion Equations in $H_{0}^{1}$
}

\author{
Yongjun Li, Yanhong Zhang, Xiaona Wei \\ School of Mathematics, Lanzhou City University, Lanzhou, China \\ Email: li liyong120@163.com
}

Received 1 March 2015; accepted 23 June 2015; published 30 June 2015

\begin{abstract}
Under the assumption that $g(t)$ is translation bounded in $L_{l o c}^{4}\left(R ; L^{4}(\Omega)\right)$, and using the method developed in [3], we prove the existence of pullback exponential attractors in $H_{0}^{1}(\Omega)$ for nonlinear reaction diffusion equation with polynomial growth nonlinearity ( $p \geq 2$ is arbitrary).
\end{abstract}

Keywords

Dynamical System, Pullback Exponential Attractors, Reaction Diffusion Equation

\section{Introduction}

Attractor's theory is very important to describe the long time behavior of dissipative dynamical systems generated by evolution equations, and there are several kinds of attractors. In this article, we will study the existence of pullback exponential attractors (see [1]-[3]) for nonlinear reaction diffusion equation. This equation is written in the following form:

$$
\left\{\begin{array}{l}
\frac{\partial u}{\partial t}-\Delta u+f(u)=g(t), x \in \Omega, \\
\left.u\right|_{\Omega}=0 \\
u(\tau)=u_{\tau .}
\end{array}\right.
$$

where $\Omega$ is a bounded smooth domain in $R^{n}, g(\cdot) \in L_{l o c}^{2}\left(R, L^{2}(\Omega)\right), f \in C^{1}(R, R)$ and there exist $p \geq 2$, $c_{i}>0, i=1,2, \cdots 5, l>0$ such that

$$
c_{1}|u|^{p}-c_{2} \leq f(u) u \leq c_{3}|u|^{p}+c_{4}, f^{\prime}(u) \geq-l,\left|f^{\prime}(u)\right| \leq c_{5}\left(1+|u|^{p-2}\right)
$$

for all $u \in R$.

How to cite this paper: Li, Y.J., Zhang, Y.H. and Wei, X.N. (2015) Pullback Exponential Attractors for Nonautonomous Reaction Diffusion Equations in $H_{0}^{1}$. Journal of Applied Mathematics and Physics, 3, 730-736. 
The Equation of (1.1) has been widely studied. For the autonomous case, i.e., $g(t)$ does not depend on the time, the asymptotic behaviors of the solution have been studied extensively in the framework of global attractor, see [4]-[6]. For the nonautonomous case, the asymptotic behaviors of the solution have been studied in the framework of pullback attractor, see [7]-[9]. Recently, the theory of pullback exponential attractor have been developed, see [1]-[3], and some methods are given to prove the existence of pullback exponential attractors.

In order to obtain the existence of pullback exponential attractors of (1.1), we will need the following theorem.

Theorem 1.1. ([3]) Let $X$ be an uniformly convex Banach space, $B(X)$ be the set of all bounded subsets of $X,\{U(t, \tau) \mid t \geq \tau\}$ be a time continuous process in $X$. Then the process $\{U(t, \tau) \mid t \geq \tau\}$ exist pullback exponential attractors in $X$ if the following conditions hold true:

(1) There exists an uniformly bounded absorbing set $B \subset X$, that is, for any $t \geq \tau$ and $D \in B(X)$, there exists $T_{0}>0$ such that

$$
U(t, \tau-s) D \subset B, \forall s \leq T_{0}
$$

(2) There exist $\delta, \theta,>0, \delta+\theta<1, T_{1}, l>0$, and a finite dimension subspace $X_{1} \subset X$, such that

$$
\begin{gathered}
\left\|U(t, \tau) u_{1}-U(t, \tau) u_{2}\right\| \leq l\left\|u_{1}-u_{2}\right\|, \forall t, \tau \in\left[k T_{1},(k+1) T_{1}\right], \forall k \in \mathbb{Z}, \\
\left\|\left(I-P_{m}\right)\left(U\left(t, t-T_{1}\right) u_{1}-U\left(t, t-T_{1}\right) u_{2}\right)\right\| \leq \delta\left\|u_{1}-u_{2}\right\|, \\
\left\|\left(I-P_{m}\right) \bigcup_{s \leq T_{1}} U(t, \tau-s) u\right\| \leq \theta, \forall t \geq \tau,
\end{gathered}
$$

for all $u, u_{1}, u_{2} \in B$ and $t \in R$, where $\delta$ is independent on the choice of $t$, and $\|\cdot\|$ is the norm in $X, I$ is the identity operator, $P_{m}: X \rightarrow X_{1}$ is a bounded projector, $m$ is the dimension of $X_{1}$.

\section{Some Estimates of Equation (1.1)}

In this section, we will derive some priori estimates for the solutions of (1.1) that will be used to construct pullback exponential attractors for the problem (1.1).

For convenience, hereafter let $|\cdot|_{p}$ be the norm of $L^{p}(\Omega)(p>1)$, and $c$ an arbitrary constant, which may difference from line to line and even in the same line. We define $H=L^{2}(\Omega)$ with scalar product $(\cdot)$ and norm $|\cdot|$; let $((\cdot))$ and $\|\cdot\|$ denote the scalar product and norm of $H_{0}^{1}(\Omega)$ and $((u, v))=\int_{\Omega} \nabla u \nabla v d x$ for all $u, v \in H_{0}^{1}(\Omega)$, set $\lambda$ is the first eigenvalue of $-\Delta$.

For the initial value problem (1.1), we know from [4]-[6] that for any initial datum $u_{0} \in H$, there exists a unique solution $u(t) \in C([\tau, T] ; H) \bigcap L^{2}\left([\tau, T] ; H_{0}^{1}\right)$ for any $T>\tau$.

Thanks to the existence theorem, the initial value problem is equivalent to a process $\{U(t, \tau) \mid t \geq \tau\}$ define by

$$
U(t, \tau): H \rightarrow H_{0}^{1}
$$

In addition, we assume that the function $g(t)$ is translation bounded in $L_{l o c}^{3}\left(R ; L^{3}(\Omega)\right)$, that is

$$
\sup _{t \in R} \int_{t}^{t+1}|g(s)|_{3}^{3} d s<\infty .
$$

By (2.1), for $\lambda>0$, we have

$$
\sup _{t \in R} \int_{t}^{t+1}|g(s)|^{2} d s<c, e^{-\lambda t} \int_{\tau}^{t} e^{\lambda s}|g(s)|^{2} d x \leq \sum_{n=0}^{\infty} \int_{t-n-1}^{t-n} e^{-\lambda(t-s)}|g(s)|^{2} d s \leq c .
$$

Lemma 2.1. ([7]-[9]) Assume that $f$ and $g$ satisfy (1.2) and (2.2), $u(t)$ be a weak solution of (1.1), then for any $t \geq \tau$, we have the following inequality:

$$
|u(t)|^{2} \leq e^{-\lambda(t-s)}\left|u_{\tau}\right|^{2}+c
$$

and

$$
\int_{\tau}^{t} e^{\lambda s}\left(\|u(s)\|^{2}+2 c_{1}|u(s)|_{p}^{p}\right) d s \leq(1+\lambda(t-\tau)) e^{\lambda \tau}\left|u_{\tau}\right|^{2}+c e^{\lambda t}+\lambda^{-1} \int_{\tau}^{t} e^{\lambda s}|g(s)|^{2} d s
$$


Lemma 2.2. Assume that $f$ and $g$ satisfy (1.2) and (2.2), $u(t)$ be a weak solution of (1.1), then the following inequality holds for $t>\tau$

$$
|u(t)|^{2}+\|u(t)\|^{2}+|u(t)|_{p}^{p} \leq c\left(1+\frac{1}{t-\tau}\right)(1+t-\tau) e^{-\lambda(t-\tau)}\left|u_{\tau}\right|^{2}+c\left(1+\frac{1}{t-\tau}\right)
$$

Obviously, for any bounded $D \subset H$, there exist $T, r>0$, such that

$$
\left|U(t, \tau) u_{\tau}\right|^{2}+\left\|U(t, \tau) u_{\tau}\right\|^{2}+\left|U(t, \tau) u_{\tau}\right|_{p}^{p} \leq r \text { for any } u_{\tau} \in D \text { and } t-\tau \geq T .
$$

Proof. Let $F(s)=\int_{0}^{s} f(\tau) d \tau$, then by (1.2), we get there exist $\tilde{c}_{i}>0, \quad i=1,2,3,4$, such that

$$
\widetilde{c_{1}}|u|^{p}-\widetilde{c_{2}} \leq F(s) \leq \widetilde{c_{3}}|u|^{p}+\widetilde{c_{4}} .
$$

Taking inner product of (1.1) with $u$ in $H$ and using (2.7), we get

$$
\frac{d}{d t}|u|^{2}+\|u\|^{2}+c \int_{\Omega} F(u) d x \leq c\left(1+|g(t)|^{2}\right)
$$

Multiply (1.1) by $u_{t}$, we have

$$
\left|u_{t}\right|^{2}+\frac{1}{2} \frac{d}{d t}\left(\|u\|^{2}+2 \int_{\Omega} F(u) d x\right)=\left(g(t), u_{t}\right),
$$

since $\left|\left(g(t), u_{t}\right)\right| \leq \frac{1}{2}\left(|g(t)|^{2}+\left|u_{t}\right|^{2}\right)$, we obtain

$$
\frac{d}{d t}\left(\|u\|^{2}+2 \int_{\Omega} F(u) d x\right) \leq|g(t)|^{2} .
$$

Combining (2.7), we get

$$
\frac{d}{d t}\left(|u|^{2}+\|u\|^{2}+2 \int_{\Omega} F(u) d x\right)+\|u\|^{2}+c \int_{\Omega} F(u) d x \leq c\left(1+|g(t)|^{2}\right) .
$$

Thanks to Poincaré inequality $\|u\| \geq \lambda|u|$, we have

$$
\|u\|^{2}+c \int_{\Omega} F(u) d x \geq \frac{\lambda}{2}|u|^{2}+\frac{1}{2}\|u\|^{2}+c \int_{\Omega} F(u) d x \geq c\left(|u|^{2}+\|u\|^{2}+c \int_{\Omega} F(u) d x\right) .
$$

Let $G(u)=|u|^{2}+\|u\|^{2}+c \int_{\Omega} F(u) d x$, by (2.9) and (2.10), we obtain

$$
\frac{d}{d t} G(u)+c G(u) \leq c\left(1+|g(t)|^{2}\right)
$$

which imply

$$
\frac{d}{d t}\left((t-\tau) e^{\lambda t} G(u)\right) \leq(1+(\lambda-c)(t-\tau)) G(u) e^{\lambda t}+c\left(1+|g(t)|^{2}\right)(t-\tau) e^{\lambda t},
$$

integrating, we get

$$
(t-\tau) e^{\lambda t} G(u) \leq(1+c(t-\tau)) \int_{\tau}^{t} G(u) e^{\lambda s} d s+c(t-\tau) e^{\lambda t}+c(t-\tau) \int_{\tau}^{t}|g(s)|^{2} e^{\lambda s} d s,
$$

using (2.3) and (2.4), we get the inequality (2.5).

Lemma 2.3. Assume that $f$ and $g$ satisfy (1.2) and (2.1), $u(t)$ be a weak solution of (1.1), then the following inequality holds for $t>\tau_{0}$

$$
|u(t)|_{2 p-2}^{2 p-2} \leq c\left(1+\frac{1}{t-\tau_{0}}\right)\left(1+t-\tau_{0}\right) e^{-\lambda\left(t-\tau_{0}\right)}\left|u_{\tau_{0}}\right|^{2}+e^{-\lambda\left(t-\tau_{0}\right)}\left|u_{\tau_{0}}\right|_{p}^{p},
$$

Here $u(t)=U(t, \tau) u_{\tau}, u_{\tau_{0}}=U\left(\tau_{0}, \tau\right) u_{\tau}$ for any $t>\tau_{0}>\tau$. 
By the assumption (2.1) and for $\lambda>0$, we get

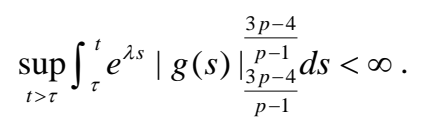

Proof. Multiply (1.1) with $|u|^{p-2} u$, we obtain

$$
\frac{1}{p} \frac{d}{d t}|u|_{p}^{p}+(p-1) \int_{\Omega}|u|^{(p-2)}|\nabla u|^{2} d x+\int_{\Omega} f(u)|u|^{p-2} u d x=\int_{\Omega} g(t)|u|^{(p-2)} u d x .
$$

By (1.2) and Young's inequality, we have

$$
f(u)|u|^{p-2} u \geq c_{1}^{\prime}|u|^{2 p-2}-c_{2}^{\prime},\left.\left.\quad\left|\int_{\Omega} g(t)\right| u\right|^{p-2} u d x\left|\leq \frac{c_{1}^{\prime}}{2}\right| u\right|_{2 p-2} ^{2 p-2}+\frac{1}{2 c_{1}^{\prime}}|g(t)|^{2} .
$$

By (2.13), we get

$$
\frac{d}{d t}|u|_{p}^{p}+c|u|_{2 p-2}^{2 p-2} \leq c\left(1+|g(t)|^{2}\right), \frac{d}{d t}\left(e^{\lambda t}|u|_{p}^{p}\right)+c e^{\lambda t}|u|_{2 p-2}^{2 p-2} \leq c e^{\lambda t}\left(1+|u|_{p}^{p}+|g(t)|^{2}\right),
$$

integrating and using (2.4), we get

$$
\int_{\tau_{0}}^{t} e^{\lambda s}|u|_{2 p-2}^{2 p-2} d s \leq c\left(\left(1+t-\tau_{0}\right) e^{\lambda \tau_{0}}\left|u_{\tau_{0}}\right|^{2}+e^{\lambda \tau_{0}}\left|u_{\tau_{0}}\right|_{p}^{p}+e^{\lambda t}+\int_{\tau_{0}}^{t} e^{\lambda s}|g(s)|^{2} d s .\right.
$$

Multiply (1.1) with $|u|^{2 p-4} u$, we obtain

$$
\frac{1}{2 p-2} \frac{d}{d t}|u|_{2 p-2}^{2 p-2}+(2 p-3) \int_{\Omega}|u|^{2 p-4}|\nabla u|^{2} d x+\int_{\Omega} f(u)|u|^{2 p-4} u d x=\int_{\Omega} g(t)|u|^{2 p-4} u d x .
$$

By (2.1), we get

$$
f(u)|u|^{2 p-4} u \geq c_{1}^{\prime \prime}|u|^{3 p-4}-c_{2}^{\prime \prime}
$$

Using Young's inequality

$$
\left.\left.\left|\int_{\Omega} g(t)\right| u\right|^{2 p-4} u d x\left|\leq \frac{c_{1}^{\prime \prime}}{2}\right| u\right|_{3 p-4} ^{3 p-4}+c \mid g(t) \frac{\left.\right|_{\frac{3 p-4}{p-1}} ^{\frac{3 p-4}{p-1}}}{{ }^{p}} .
$$

By the above inequality, we have

$$
\frac{d}{d t}|u|_{2 p-2}^{2 p-2} \leq c\left(1+\mid g(t) \frac{\left.\right|_{\frac{3 p-4}{p-1}} ^{p-1}}{p-1}\right), \frac{d}{d t}\left(\left(t-\tau_{0}\right) e^{\lambda t}|u|_{2 p-2}^{2 p-2}\right) \leq\left(1+\lambda\left(t-\tau_{0}\right) e^{\lambda t}|u|_{2 p-2}^{2 p-2}+c\left(t-\tau_{0}\right) e^{\lambda t}\left(1+|g(t)|_{\frac{3 p-4}{p-1}}^{\frac{3 p-4}{p-1}}\right),\right.
$$

integrating and using (2.12) and (2.14), we get (2.11) holds.

Lemma 2.1, lemma 2.2 and lemma 2.3 show that the process generated by the equation (1.1) have an uniformly pullback bounded absorbing set in $H, H_{0}^{1}, L^{p}(\Omega), L^{2 p-2}(\Omega)$, that is

Theorem 2.4. Assume that $f$ and $g$ satisfy (1.2) and (2.1), $u(t)$ be a weak solution of (1.1), then the process generated by the equation (1.1) have an uniformly pullback bounded absorbing set

$B \subset H \cap H_{0}^{1} \cap L^{p}(\Omega) \cap L^{2 p-2}(\Omega)$, that is, for any bounded set $D \subset H$, there exists $T_{0}>0$, such that $U(t, t-\tau) D \subset B$ for any $t-\tau \geq T_{0}$.

In fact, using the same proof as in Lemma 2.3, we can get the following result.

Lemma 2.5. Assume that $f$ satisfies (1.2), $g(t)$ is translation bounded in $L_{l o c}^{4}\left(R ; L^{4}(\Omega)\right)$, that is $\sup _{t \in R} \int_{t}^{t+1}|g(s)|_{4}^{4} d s<\infty, \quad u(t)$ be a weak solution of (1.1), then the process generated by the equation (1.1) have an uniformly pullback bounded absorbing set $B \subset L^{4 p-6}(\Omega)$, that is, for any bounded set $D \subset H$, there exists $T_{0}>0$, such that $U(t, t-\tau) D \subset B$ for any $t-\tau \geq T_{0}$.

\section{Pullback Exponential Attractors}

In this section, we will use Theorem 1.1 to prove that the process generated by Equation (1.1) exists a pullback 
exponential attractor.

First we assume that the function $g(t)$ is normal ([10]) in $L_{l o c}^{2}(R, H)$, that is, for any $\varepsilon>0$, there exists $\eta>0$ such that

$$
\sup _{t \in R} \int_{t}^{t+\eta}|g(s)|^{2} d s<\varepsilon \text {. }
$$

Obviously, $g(t)$ is normal in $L_{l o c}^{2}(R, H)$ implying that $g(t)$ is translation bounded in $L_{l o c}^{2}(R, H)$.

We set $A=-\Delta$, since $A^{-1}$ is a continuous compact operator in $H$, by the classical spectral theorem, there exist a sequence $\left\{\lambda_{j}\right\}_{j=1}^{\infty}, \quad 0<\lambda_{1} \leq \lambda_{2} \leq \cdots \leq \lambda_{j} \leq \cdots, \lambda_{j} \rightarrow+\infty a s j \rightarrow+\infty$, and a family of elements $\left\{e_{j}\right\}_{j=1}^{\infty}$ of $H_{0}^{1}$ which are orthogonal in $H$ such that $A e_{j}=\lambda_{j} e_{j}, \forall j \in \mathbb{Z}^{+}$. Let $H_{m}=\operatorname{span}\left\{e_{1}, e_{2}, \cdots, e_{m}\right\}$ in $H$ and $P: H \rightarrow H_{m}$ is a orthogonal projector. For any $u \in H$, we write

$$
u=P u+(I-P) u \triangleq u_{1}+u_{2} .
$$

Theorem 2.4. Assume that $f$ satisfies (1.2), $g(t)$ is translation bounded in $L_{\text {loc }}^{4}\left(R ; L^{4}(\Omega)\right)$ and (3.1) holds, then the process generated by the equation (1.1) have a pullback exponential attractor.

Next, we will verify that the process generated by (1.1) satisfy all the conditions of Theorem 1.1.

Proof. By Theorem 2.4, there exists $T_{0}>0$, such that $U(t, t-\tau) B \subset B$ for any $t-\tau \geq T_{0}$. Let $B^{\prime}=\cup_{t \in R} \cup_{\tau \leq T_{0}} U(t, t-\tau) B$, we obtain $B^{\prime}$ is also an uniformly pullback bounded absorbing set in $H_{0}^{1} \cap L^{2 p-2}(\Omega)$ and $U(t, t-\tau) B^{\prime} \subset B^{\prime}$ for any $t \geq \tau$.

We set $u_{1}(t)=U(t, \tau) u_{1 \tau}, \quad u_{2}(t)=U(t, \tau) u_{2 \tau}$ to be solutions associated with Equation (1.1) with initial data $u_{1 \tau}, u_{2 \tau} \in B^{\prime}$, since $B^{\prime}$ is the uniformly pullback bounded absorbing set in $H_{0}^{1} \cap L^{2 p-2}(\Omega)$, so there exists $M>0$ such that $\left\|u_{i \tau}\right\| \leq M,\left\|u_{i}(t)\right\| \leq M,\left|u_{i}(t)\right|_{2 p-2}^{2 p-2} \leq M, \quad i=1,2$. Let $w(t)=u_{1}(t)-u_{2}(t)$, by (1.1), we get

$$
w_{t}-\Delta w+f\left(u_{1}(t)-u_{1}(t)\right)=0 .
$$

Taking inner product of (3.2) with $-\Delta w$ in $H$, we have

$$
\frac{1}{2} \frac{d}{d t}\|w\|^{2}+\|\Delta w\|^{2}+\left(f\left(u_{1}(t)-u_{1}(t)\right),-\Delta w\right)=0 .
$$

Taking into account (1.2) and Holder inequality, it is immediate to see that

$$
\left|\left(f\left(u_{1}\right)-f\left(u_{2}\right),-\Delta w\right)\right| \leq \int_{\Omega}\left|f\left(u_{1}\right)-f\left(u_{2}\right)\right||\Delta w| d x \leq \frac{1}{2}|\Delta w|^{2}+\frac{1}{2} \int_{\Omega}\left|f\left(u_{1}\right)-f\left(u_{2}\right)\right|^{2} d x,
$$

and

$$
\begin{aligned}
& \int_{\Omega}\left|f\left(u_{1}\right)-f\left(u_{2}\right)\right|^{2} d x=\int_{\Omega}\left|f^{\prime}\left(u_{1}+\theta\left(u_{2}-u_{1}\right)\right)^{2}\right| u_{2}-\left.u_{1}\right|^{2} d x \leq c \int_{\Omega}\left(1+\left|u_{1}\right|^{p-2}+\left|u_{2}\right|^{p-2}\right)^{2}\left|u_{2}-u_{1}\right|^{2} d x \\
& \leq c \int_{\Omega}\left(1+\left|u_{1}\right|^{2 p-3}+\left|u_{2}\right|^{2 p-3}\right)\left|u_{1}-u_{2}\right| d x \leq c\left(1+\left|u_{1}\right|_{4 p-6}^{4 p-6}+\left|u_{2}\right|_{4 p-6}^{4 p-6}\right)|w|^{2}
\end{aligned}
$$

By Lemma 2.5, we get

$$
\int_{\Omega}\left|f\left(u_{1}\right)-f\left(u_{2}\right)\right|^{2} d x \leq c\|w\|^{2}
$$

Using (3.3), we obtain $\frac{d}{d t}\|w\|^{2} \leq c\|w\|^{2}$, hence

$$
\|w(t)\|^{2} \leq\|w(\tau)\|^{2} e^{c(t-\tau)} .
$$

Let $w=w_{1}+w_{2}, w_{1}$ be the project in $P H$. Taking inner product of (3.2) with $-\Delta w_{2}$ in $H$, we have

$$
\frac{1}{2} \frac{d}{d t}\left\|w_{2}\right\|^{2}+\left|\Delta w_{2}\right|^{2}+\left(f\left(u_{1}\right)-f\left(u_{2}\right),-\Delta w_{2}\right)=0 .
$$




$$
\left|\left(f\left(u_{1}\right)-f\left(u_{2}\right),-\Delta w_{2}\right)\right| \leq \int_{\Omega}\left|f\left(u_{1}\right)-f\left(u_{2}\right)\right|\left|\Delta w_{2}\right| d x \leq \frac{1}{2}\left|\Delta w_{2}\right|^{2}+\frac{1}{2} \int_{\Omega}\left|f\left(u_{1}\right)-f\left(u_{2}\right)\right|^{2} d x .
$$

Taking into (3.4) account, we obtain

$$
\frac{d}{d t}\left\|w_{2}\right\|^{2}+\left|\Delta w_{2}\right|^{2} \leq c\|w\|^{2},
$$

Using the Poincaré inequality $\lambda_{n}\left\|w_{2}\right\|^{2} \leq\left|\Delta w_{2}\right|^{2}$, we get $\frac{d}{d t}\left\|w_{2}\right\|^{2}+\lambda_{n}\left\|w_{2}\right\|^{2} \leq c\|w\|^{2}$, by Gronwall's Lemma, we have $\left\|w_{2}(t)\right\|^{2} \leq e^{-\lambda_{n}(t-\tau)}\|w(\tau)\|^{2}+c e^{-\lambda_{n} t} \int_{\tau}^{t} e^{-\lambda_{n} s}\|w(s)\|^{2} d s$. Using (3.5), we get

$$
\left\|w_{2}(t)\right\|^{2} \leq\left(e^{-\lambda_{n}(t-\tau)}+c \frac{e^{c(t-\tau)}}{\lambda_{n}}\right)\|w(\tau)\|^{2} .
$$

Let $u(t)=u_{1}(t)+u_{2}(t), \quad u_{1}(t)$ be the project in $P H$. Taking inner product of (1.1) with $-\Delta u_{2}$, we get

$$
\frac{1}{2} \frac{d}{d t}\left\|u_{2}\right\|^{2}+\left\|\Delta u_{2}\right\|^{2}+\left(f(u(t)),-\Delta u_{2}\right)=\left(g(t),-\Delta u_{2}\right) .
$$

Since $\left|\left(g(t),-\Delta u_{2}\right)\right| \leq|g(t)|^{2}+\frac{1}{4}\left|\Delta u_{2}\right|^{2}, \quad\left|\left(f(u(t)),-\Delta u_{2}\right)\right| \leq \int_{\Omega}|f(u)|^{2} d x+\frac{1}{4}\left|\Delta u_{2}\right|^{2}$, and by Poincaré inequality $\lambda_{n}\left\|u_{2}\right\|^{2} \leq\left|\Delta u_{2}\right|^{2}$, we have

$$
\frac{d}{d t}\left\|u_{2}\right\|^{2}+\lambda_{n}\left\|u_{2}\right\|^{2} \leq c\left(1+|g(t)|^{2}\right)
$$

By Gronwall’s lemma, we get

$$
\left|u_{2}(t)\right|^{2} \leq e^{-\lambda_{n}(t-\tau)}\left|u_{\tau}\right|^{2}+c e^{-\lambda_{n} t} \int_{\tau}^{t} e^{\lambda_{n} s}\left(1+|g(s)|^{2}\right) d s .
$$

By (3.1), we obtain that there exists $c>0$, such that $\int_{t}^{t+1}|g(s)|^{2} d s<c$ for any $t \in R$, and for any $\varepsilon>0$, there exists $\eta>0$, such that $\int_{t-\eta}^{t}|g(s)|^{2} d s<\frac{\varepsilon}{3}$, so we get

$$
\begin{aligned}
& e^{-\lambda_{n} t} \int_{\tau}^{t} e^{-\lambda_{n} s}|g(s)|^{2} d s=\int_{\tau}^{t} e^{-\lambda_{n}(t-s)}|g(s)|^{2} d s \\
\leq & \int_{t-\eta}^{t} e^{-\lambda_{n}(t-s)}|g(s)|^{2} d s+\int_{t-\eta-1}^{t-\eta} e^{-\lambda_{n}(t-s)}|g(s)|^{2} d s \\
& +\int_{t-\eta-2}^{t-\eta-1} e^{-\lambda_{n}(t-s)}|g(s)|^{2} d s+\cdots+\int_{t-\eta-k-1}^{t-\eta-k} e^{-\lambda_{n}(t-s)}|g(s)|^{2} d s+\cdots \\
\leq & \frac{\varepsilon}{3}+c e^{-\lambda_{n} \eta}\left(1+e^{-\lambda_{n}}+e^{-2 \lambda_{n}}+\cdots+e^{-k \lambda_{n}}+\cdots\right) \leq \frac{\varepsilon}{3}+\frac{e^{-\lambda_{n} \eta}}{1-e^{-\lambda_{n}}} \\
& \text { and } e^{-\lambda_{n} t} \int_{\tau}^{t} e^{\lambda_{n} s} d s \leq \frac{1}{\lambda_{n}} \text {, we have }\left.\right|^{2} \leq e^{-\lambda_{n}(t-\tau)}\left|u_{\tau}\right|^{2}+c\left(\frac{1}{\lambda_{n}}+\frac{\varepsilon}{3}+\frac{e^{-\lambda_{n} \eta}}{1-e^{-\lambda_{n}}}\right) .
\end{aligned}
$$

Let $T_{1}=t-\tau=1$, by (3.5), we get

$$
\left\|U(t, \tau) u_{1 \tau}-U(t, \tau) u_{2 \tau}\right\| \leq e^{c}\left\|u_{1 \tau}-u_{2 \tau}\right\| .
$$

Since $\lambda_{n} \rightarrow+\infty$, for $0<\varepsilon<1$, from (3.7) and (3.8), there exist $m \in \mathbb{Z}^{+}, \delta, \theta,>0, \delta+\theta<1$ such that

$$
\begin{gathered}
\left\|\left(I-P_{m}\right)\left(U\left(t, t-T_{1}\right) u_{1}-U\left(t, t-T_{1}\right) u_{2}\right)\right\| \leq \delta\left\|u_{1}-u_{2}\right\|, \\
\left\|\left(I-P_{m}\right) \bigcup_{s \leq T_{1}} U(t, \tau-s) u\right\| \leq \theta, \forall t \geq \tau .
\end{gathered}
$$


By Theorem 2.4 and (3.9)-(3.11), we know that the process $\{U(t, \tau) \mid t \geq \tau\}$ generated by (1.1) satisfy all the conditions of Theorem 1.1 .

\section{Funds}

This work was supported by the National Nature Science Foundation of China (11261027) and Longyuan youth innovative talents support programs of 2014, and the innovation Funds of principal (LZCU-XZ2014-05).

\section{References}

[1] Langa, J., Miranville, A. and Real, J. (2010) Pullback Exponential Attractors. Discrete and Continuous Dynamical Systems-Series A, 26, 1329-1357.

[2] Czaja, R. and Efendiev, M. (2011) Pullback Exponential Attractors for Non-Autonomous Equations, Part I: Semilinear parabolic Problems. Journal of Mathematical Analysis and Applications, 381, 748-765. http://dx.doi.org/10.1016/j.jmaa.2011.03.053

[3] Li, Y., Wang, S. and Zhao, T. (2015) The Existence of Pullback Exponential Attractors for Non-Autonomous Dynamical System and Application to Non-Autonomous Reaction Diffusion Equations. J. Appl. Anal. Comp (in press).

[4] Chepyzhov, V. and Vishik, M. (2002) Attractors for Equations of Mathematics Physics. 49, American Mathematical Society Colloquium Publications, AMS.

[5] Temam, R. (1997) Infinite-Dimensional Dynamical Systems in Mechanics and Physics. 68, Springer, New York. http://dx.doi.org/10.1007/978-1-4612-0645-3

[6] Ladyzhenskaya, O. (1991)Attractors for Semigroups and Evolution Equations. Cambridge University Press, Cambridge, UK. http://dx.doi.org/10.1017/CBO9780511569418

[7] Song, H. and Wu, H. (2007) Pullback Attractor for Nonlinear Autonomous Reaction Diffusion Equations. Journal of Mathematical Analysis and Applications, 325, 1200-1215. http://dx.doi.org/10.1016/j.jmaa.2006.02.041

[8] Song, H. (2010) Pullback Attractors of Nonautonomous Reaction Diffusion Equation in $H_{0}^{1}$. Journal of Differential Equations, 249, 2357-2376. http://dx.doi.org/10.1016/j.jde.2010.07.034

[9] Li, Y. and Zhong, C. (2007) Pullback Attractors for the Norm-to-Weak Continuous Process and Application to the Non-Autonomous Reaction Diffusion Equations. Appl. Math. Comp., 190, 1020-1029. http://dx.doi.org/10.1016/j.amc.2006.11.187

[10] Lu, S., Wu, Q. and Zhong, C. (2005) Attractors for Nonautonomous 2D Navier-Stokes Equation with Normal External Forces. Discrete Cont. Dyna. Syst, 13, 701-719. http://dx.doi.org/10.3934/dcds.2005.13.701 